\title{
MODELS OF PROCESSES AS COMPONENTS OF AIR PASSENGER FLOW MODEL
}

The paper presents the model of air passenger processes at airports for single source and two source processes. It shall be used as a component for the development of passenger flow model. In the paper the single source model is applied on passenger check-in process. It is based on deterministic queuing model where cumulative diagrams of the flow are used for calculations. As input data the model requires the arrival and service profile of passengers at the check-in. The arrival profile is a cumulative number of passengers arriving to the server during the time. The service profile is a cumulative number of passengers which can be served by the check-in process. The calculation of average waiting time and average length of the waiting queue is presented in the paper. In latter part of the paper the case of two source process is discussed.

Key words: Air passenger flow model, airport passenger processes, deterministic queuing, cumulative diagrams

\section{Introduction}

The context of our research is the evaluation of future airport concepts in which the airport airsides and the landsides are separated. This idea was presented in [1] and further elaborated in [2]. For the evaluation of the concepts we decided to develop a model of Airside-Landside Separated airports (ASLS). The model should simulate air passenger movements from the city air terminal (landside terminal) to the airside gates while passing through standard passenger-related airport processes such as the check-in, the security, etc. In the literature models do exist for passenger flows at airports. The queuing theory based model presented in [3] is oriented on passenger waiting times in an air terminal based on passenger arrivals in relation to the capacity of passenger processors (i.e. check-in, passport control, departure lounge and embarkation). This queuing theory based approach is too constraining for the development of our model since it requires strong assumptions such as the steady arrival rate. Thus it does not capture well the dynamics of passenger arrivals. On a microscopic level [4], [5], [6] developed the simulation model in ARENA simulation software. An analytical aggregate model for estimating capacity and delays at airport terminals has been presented in [7]. Neither of above mentioned models does capture passenger movement or passenger processes prior to their arrival to the terminal. The models in the articles have not been described in the detail, and often processes have been pre-programmed in the simulation software. Thus it is not transparent for us how the passenger process has been modelled in the detail. For these reasons we decided to develop our own model based on the knowledge about the passenger behaviour. We classified the air passenger activities into passenger processes and passenger movements among the processes.
To get an overview of which models exist that are able to represent passenger processes and movements we conducted a literature review. For the passenger movements the identified models where classified based on the modelling approach to mircosimulation models, cellular automata models, queuing theory based models, gas-kinetics based models and continuum based models, whereas this classification was adopted from [8]. For passenger processes the modelling approaches identified where stochastic models based on queuing theory [3], deterministic models using cumulative diagrams [9], [10] and simulation models [11]. This paper focuses on the processes while the key question is what modelling approaches would best fit for the development of our passenger flow model. The discussion about existing models and the prototype of our model for processes based on the deterministic queuing model is presented.

\section{Modelling of Passenger Processes at Airports}

\subsection{Single source processes}

For the passenger processes we considered three options as it is mentioned in the introduction: queuing theory based stochastic models, deterministic queuing models and microsimulation models. In our case the queuing theory models require too unrealistic assumptions about passenger behaviour. For example it requires constant passenger arrival rate while at airports passengers often arrive at variable rate according to the so called arrival profile mentioned in [12]. On the other hand the microsimulation can capture individual characteristics of a single passenger. However, it often requires large amount of data as an input especially about

\footnotetext{
* Martin Matas ${ }^{1}$, Andrej Novak

Department of Air Transport, Faculty of Operation and Economics of Transport and Communications, University of Zilina, Slovakia,

E-mail: Martin.Matas@fpedas.uniza.sk

${ }^{1}$ Martin Matas, $\mathrm{PhD}$ student at University of Zilina, realising the PhD study under co-supervision with Eurocontrol Experimental Centre, Bretigny sur Orge, France
} 
the passengers and the airport layout. The model may become too large in terms of input data required and too complex in terms of an amount of objects in it. Therefore for the modelling of the processes we decided to use deterministic queuing model based on cumulative diagrams. This approach is at a mesoscopic level of detail, because it can not represent individual passenger behaviour as microscopic model could but, on the other hand, it still can capture the characteristics of a group of passengers in terms of their arrival behaviour as an advantage against too rigid stochastic queuing models. The model requires that the passenger arrival profile and the server service profile are known. It could be represented by $A(t)$ and $S(t)$ functions for arrival and service profile respectively. These functions are used to produce departure profile $D(t)$ representing passenger departures from the server. By using $A(t)$ and $D(t)$ functions other operational variables could be calculated. Every passenger waits in the line certain time ranging from zero to some value. Sum of all waiting times could be calculated as an area bounded between $A(t)$ and $D(t)$ functions:

$$
T_{\text {wait }}=\int(A(t)-D(t)) d t
$$

Cumulative number of passengers at the time $t$ is represented by $N(t)$. Then the average waiting time per passenger until the time $t$ is:

$$
t_{\text {wait_avg }}=\frac{T_{\text {wait }}}{N(t)}=\frac{\int(A(t)-D(t)) d t}{N(t)}
$$

Analogically, the average length of the queue as an area bounded between $A(t)$ and $D(t)$ divided by elapsed time between the arrival of the first passenger $\left(t_{0}\right)$ and the facility(e.g. check-in) closing time $\left(t_{\text {close }}\right)$ :

$$
n_{\text {wait_avg }}=\frac{T_{\text {wait }}}{\left(t_{0}-t_{\text {close }}\right)}=\frac{\int(A(t)-D(t)) d t}{\left(t_{0}-t_{\text {close }}\right)}
$$

Other queuing operational characteristics could be calculated but these two are of our particular interest since passenger waiting time could be used for calculating the total travel time and the length of the queue could be used for determining the space capacity of the facility area.

Based on the above mentioned modelling approach we developed a prototype of the passenger process model with a working name "PaxMod". At this stage of development the model requires the arrival and departure profiles to be stepwise linear. Therefore it takes as an input the set of integer data representing the cumulative number of passengers arriving and departing from the server during the time. An example of such data input is presented in Table 1 and Table 2. The data from the tables were collected at Brno-Turany international airport in the Czech Republic on Sunday $20^{\text {th }}$ of May 2007 on the scheduled flight of Ryanair to London Stansted airport. The data inputs are then visualised in a discrete cumulative diagram as it is depicted on the upper part of Fig 1.
CUMULATIVE NUMBER OF PASSENGERS

Table 1 ARRIVING TO THE CHECK-IN AT A GIVEN TIME

\begin{tabular}{|c|c|}
\hline Time from STD $(\mathrm{min})$ & Cumulative arrivals (pax) \\
\hline-200 & 0 \\
\hline-190 & 4 \\
\hline-170 & 7 \\
\hline-160 & 8 \\
\hline-140 & 15 \\
\hline-130 & 18 \\
\hline-120 & 40 \\
\hline-100 & 65 \\
\hline-90 & 107 \\
\hline-70 & 124 \\
\hline-60 & 162 \\
\hline-40 & 179 \\
\hline-30 & 180 \\
\hline-10 & 184 \\
\hline 0 & 184 \\
\hline
\end{tabular}

CUMULATIVE NUMBER OF PASSENGERS Table 2 DEPARTING FROM THE CHECK-IN AT A GIVEN TIME

\begin{tabular}{|c|c|}
\hline Time from STD $(\mathrm{min})$ & Cumulative departures (pax) \\
\hline-140 & 0 \\
\hline-120 & 12 \\
\hline-100 & 50 \\
\hline-90 & 84 \\
\hline-70 & 119 \\
\hline-60 & 156 \\
\hline-40 & 184 \\
\hline-30 & 184 \\
\hline-10 & 184 \\
\hline 0 & 184 \\
\hline
\end{tabular}

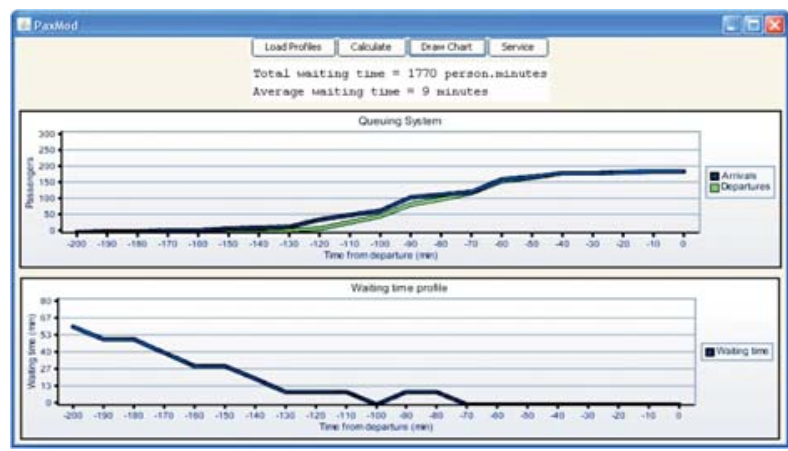

Fig 1 Evolution of passenger arrivals, departures and waiting times at the Check-in (screen shot from PaxMod). 


\subsection{Processes with two sources}

The above mentioned model can represent the evolution of passenger arrivals and departures from the server however it can neither handle characteristics of individual passenger nor a group of passengers. As it has been mentioned earlier we are not interested in individual passengers, however, we are still interested in a group of passengers especially in terms of their waiting times. For instance, we need to distinguish between business and leisure passengers. Moreover the passengers may arrive from different sources. For example, there might be transit passengers arriving to the gate together with departing passengers thus creating one single arrival profile to the gate. To be able to distinguish between the two or more groups we chose an approach explained in the following paragraphs.

Let us suppose that there are two different groups of passengers arriving to the server. One group would be represented by the letter $A$ and the other one by the letter $B$ the frequency of arrivals of the passengers from the groups $A$ and $B$ is represented by the functions $a(t)$ and $b(t)$. Arrival profiles for both groups are integrals of the arrival-frequency functions:

$$
\begin{aligned}
& A(t)=\int_{t_{0}}^{t} a(\tau) d \tau \\
& B(t)=\int_{t_{0}}^{t} b(\tau) d \tau
\end{aligned}
$$

These arrival profiles are combined together in one single arrival profile

$$
C(t)=A(t)+B(t)
$$

The service profile of the server is represented by the function $S(t)$. From $C(t)$ and $S(t)$ it is possible to calculate performance variables for the mixed group consisting of the passengers $A$ and $B$. However we need to calculate these variables for each group individually. Therefore, we need to know what part of the service profile is used by the group A and what part is used by the group B. Thus we divide service profile $S(t)$ on two profiles $S A(t)$ and $S B(t)$ so that $S(t)=S A(t)+S B(t)$. $S A(t)$ and $S B(t)$ could be calculated as follows.

During the short period of time $\Delta t$ starting at $t_{i}$ the server with the service profile $S(t)$ can process $\Delta n$ passengers who stand behind the position $n_{i}$ in the waiting line (Fig 2). The service rate at which the passengers are processed is denoted by $s(t)$ where $s(t)=d S(t) / d t$. This service rate is proportionally distibuted between $A$ and $B$ passengers: $s(t)=s_{A}(t)+s_{B}(t)$. The proportion of the $A$ or $B$ passengers in the small group of $\Delta n$ passengers is denoted by $\rho_{N A}\left(n_{i}\right)$. $\Delta n$ and $\rho_{N B}\left(n_{i}\right)$. $\Delta n$ whereas $\rho_{N A}\left(n_{i}\right)$ and $\rho_{N B}\left(n_{i}\right)$ represent the proportion of $A$ or $B$ passengers in the $\Delta n$. Thus

$$
\begin{aligned}
& s_{A}\left(t_{i}\right)=\rho_{N A}\left(n_{i}\right) \cdot s\left(t_{i}\right) \\
& s_{B}\left(t_{i}\right)=\rho_{N B}\left(n_{i}\right) \cdot s\left(t_{i}\right)
\end{aligned}
$$

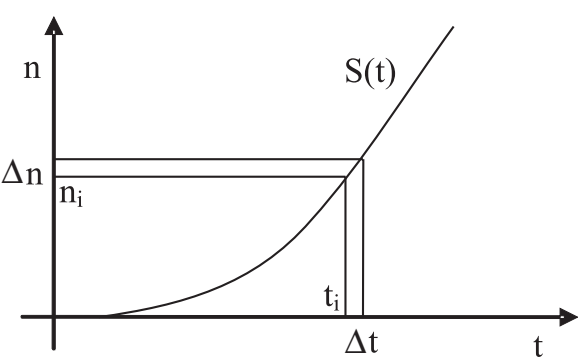

Fig 2 Example of passenger service profile as a function of time.

Now we need to calculate the $\rho_{N A}(n)$ and $\rho_{N B}(n)$ functions. They represent kind of distribution of the passengers $A$ and $B$ in the waiting line. This distribution depends on $a(t)$ and $b(t)$ arrival profiles.

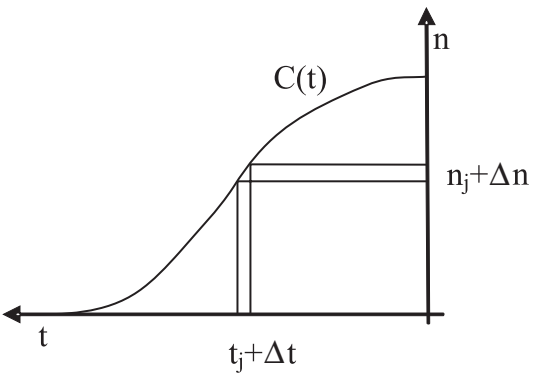

Fig 3 Example of passenger arrival profile as a function of time.

During the short period of time $\Delta t$ starting at $t_{j}$ there are $\Delta n$ passenger arrivals to the waiting line (Fig 3 ). The proportion of $A$ or $B$ passengers is dependent on the ratio between their arrival rates in a way that

$$
\begin{aligned}
& \rho_{T A}(t)=\frac{a(t)}{a(t)+b(t)} \\
& \rho_{T B}(t)=\frac{b(t)}{a(t)+b(t)}
\end{aligned}
$$

for every time moment $t$ there exist a position $n$ in the line according to the $C(t)$ where as

$$
n=C(t)
$$

To obtain time moment $t$ from (11) we need to use inverse funtion from $C(t)$ denoted by $C^{-1}(n)$ so that

$$
t=C^{-1}(n)
$$

If we use (12) as an input to our $\rho_{T A}(t)$ and $\rho_{T B}(t)$ functions we obtain passenger distribution in the waiting line:

$$
\begin{aligned}
& \rho_{N A}(n)=\rho_{T A}(t)=\rho_{T A}\left(C^{-1}(n)\right) \\
& \rho_{N B}(n)=\rho_{T B}(t)=\rho_{T B}\left(C^{-1}(n)\right)
\end{aligned}
$$


Later when the service starts $n=S(t)$ which can be put into (13) and (14) to obtain (15) and (16)

$$
\begin{aligned}
& \rho_{N A}(n)=\rho_{T A}\left(C^{-1}(S(t))\right) \\
& \rho_{N B}(n)=\rho_{T B}\left(C^{-1}(S(t))\right)
\end{aligned}
$$

To obtain a service rate for the group $A$ and $B$ we combine (7) with (15) and (8) with (16)

$$
\begin{aligned}
& s_{A}(t)=\rho_{T A}\left(C^{-1}(S(t))\right) \cdot s(t) \\
& s_{B}(t)=\rho_{T B}\left(C^{-1}(S(t))\right) \cdot s(t)(18)
\end{aligned}
$$

Service profiles are parametrical integrals of service rates:

$$
\begin{aligned}
& S_{A}(t)=\int_{t_{0}}^{t} S_{A}(\tau) d \tau \\
& S_{B}(t)=\int_{t_{0}}^{t} S_{B}(\tau) d \tau
\end{aligned}
$$

If the service time is relatively small in comparison to the waiting time we can use service profile as a departure profile. Then using $A(t), B(t), S_{A}(t)$ and $S_{B}(t)$ we can calculate performance variables for each group individually the same way as for service with only one arrival group (par. 2.1).

\section{Conclusion}

The general aim of our research is to develop a passenger flow model. This would be based on elementary models of passenger processes and passenger movements. So far we have developed a prototype of passenger process applied on the airport check-in. We chose the deterministic queuing approach based on cumulative diagrams. It does not track individual passengers, but it can capture the global performance variables such as an average waiting time, average queue length, average service rate etc. The paper presents how to divide the service profile in two service profiles if there are two sources of arrivals. It is useful when each group of passengers needs to be tracked even in case they mix with other groups in arrival queue.

\section{Future Work}

At this stage of our research we have been focusing on passenger processes for the development of the passenger flow model. As it is mentioned in the introduction we conducted literature review for existing models of passenger movements. We have not yet developed the model for movements although we have drawn following conclusions from the literature review. For the evaluation of future airport concepts we are interested in passenger flows rather than in individual behaviour of a passenger. Therefore, microscopic models such as microsimulation models or cellular automata models are too detailed and require too much data about the modelled object. On the other hand, macroscopic models may not be able to capture flow dynamics realistically since they are often based on strong assumptions which tend to be unrealistic. For these reasons we decided to make a compromise between the two extremes of having a too detailed model or too unrealistic assumptions. Passenger movements are to be modelled as a flow between the processes where the speed of the passenger flow would depend on the free flow speed and the density of passengers in the corridor. It could be represented using fundamental diagrams elaborated in [13]. The output from the processes would be the input for the models of movements and vice-versa. Therefore, the level of detail for the model of passenger movements would match the level of detail of the model used for passenger processes. Once we develop models of passenger movements at airports they, in turn, could be used as components to connect air passenger processes thus creating the basic structure of a passenger flow model.

\section{References}

[1] BROCHARD, M.: The Airport of the Future or Breaking the Constraints Between the Terminal and the Runways, Innovative Research Activity Report 2004, pp. 37-45, Eurocontrol Experimental Centre, 2004.

[2] MATAS, M.: Future Airport Concept, Activity Report 2005, pp. 83-92, Eurocontrol Experimental Centre, 2005.

[3] ASHFORD, N., HAWKINS, N., O'LEARY, M.: Passenger Behaviour and Design of Airport Terminals, Transportation Research Board Record, 588:19-26, 1976.

[4] GATERSLEBEN, M. R., VAN DER WEIJ, S. W.: Analysis and Simulation of Passenger Flows in an Airport Terminal, Proceedings of the 1999 Winter Simulation Conference, pp. 1226-1231, 1999.

[5] KIRAN, A. S., TEKIN, C., SERAFETTIN, O.: Simulation Modeling and Analysis of a New International Terminal, Proc. of the 2000 Winter Simulation Conference, pp. 1168-1172, 2000.

[6] BABELIOWSKY, M.: Designing Interorganizational Logistic Networks, A simulation based interdisciplinary approach. PhD thesis, Technische Universiteit Delft, 1997.

[7] BRUNETTA, L., RIGHI, L., ANDREATTA, G.: An Operations Research Model for the Evaluation of an Airport Terminal: SLAM (simple landside aggregate model), Journal of Air Transport Management, 3/1999, pp. 161-175(15), Publisher: Elsevier

[8] DAAMEN, W.: Modelling Passenger Flows In Public Transport Facilities, PhD thesis, Technische Universiteit Delft, 2004.

[9] DE NEUfVILLE, R., ODONI, A.: Airport Systems Planning Design and Management, pp. 134-135, McGraw-Hill, 2003.

[10] HORONJEFF, R.: Analyses of Passenger and Baggage Flows in Airport Terminal Building, Journal of Aircraft, 6(5):446-451, 1969. 


\section{COMMNICOIIIONS}

[11] JOUSTRA, P. E., VAN DIJK, N. M.: Simulation of Check-in at Airports, Proc. of 2001 Winter simulation conference, pp. 1023-1028, 2001.

[12] IATA, International Air Transport Association, Airport Terminal Reference Manual, Seventh Edition, Montreal, Canada, 1989.

[13] SEYFRIED, A., STEFFEN, B., KLINGSCH, W., BOLTES, M.: The Fundamental Diagram of Pedestrian Movement Revisited, Journal of Statistical Mechanics: Theory and Experiment, 10:10002, 2005. 